\title{
NOVEL MODELING OF HYDROGEN/OXYGEN DETONATION *
}

\author{
Yevgenii Rastigejev ${ }^{\dagger}$ Sandeep Singh $\ddagger$ Christopher Bowman ${ }^{\S}$ Samuel Paolucci, ${ }^{\Uparrow}$ and Joseph M. Powers.॥ \\ Department of Aerospace and Mechanical Engineering, \\ University of Notre Dame, \\ Notre Dame, Indiana 46556-5637.
}

\begin{abstract}
$\underline{\text { Abstract }}$
A standard ignition delay problem for a mixture of hydrogen, oxygen, and argon in a shock tube is extended to the viscous regime and solved using the method of Intrinsic Low Dimensional Manifolds (ILDM) coupled with a Wavelet Adaptive Multilevel Representation (WAMR) spatial discretization technique. An operator splitting method is used to describe the reactions as a system of ordinary differential equations at each spatial point. The ILDM method is used to eliminate the stiffness associated with the chemistry by decoupling processes which evolve on fast and slow time scales. The fast time scale processes are systematically equilibrated, thereby reducing the dimension of the phase space required to describe the reactive system. The WAMR captures the detailed spatial structures automatically with a small number of basis functions thereby further reducing the number of variables required to describe the system. Using a maximum of only $300 \mathrm{col}-$ location points and 15 scale levels allows results with striking resolution of fine scale viscous and induction zones to be obtained. Additionally, the resolution of physical diffusion processes minimizes the effects of potentially reaction-inducing artificial entropy layers associated with numerical diffusion.
\end{abstract}

\section{$\underline{\text { Introduction }}$}

This paper will present results of the application of new algorithms for scientific computing to a standard problem in combustion. The ILDM method, which rationally reduces detailed kinetic systems, and

\footnotetext{
${ }^{*}$ Copyright (C) by J. M. Powers and S. Paolucci. Published by the American Institute of Aeronautics and Astronautics, Inc., with permission. This study was supported by NSF under CTS-9705150 and AFOSR under F49620-98-1-0206.

$\dagger$ Graduate Assistant.

$\ddagger$ Graduate Assistant, Member AIAA.

$\S$ Post-Doctoral Research Associate.

व Professor.

" Associate Professor, Associate Fellow AIAA.
}

WAMR, which allows for efficient resolution of detailed spatial structures, are brought together to address the problem of ignition delay in a shock tube filled with a mixture of hydrogen, oxygen, and argon.

It is well known that in order to accurately simulate a wide variety of thermochemical phenomena, the effects of detailed finite rate chemistry must be incorporated into models. However, implementation of fully detailed chemistry models with the obligatory numerical resolution has proved to require a prohibitive amount of computational resources for all but the simplest of flows. While the addition of species and reaction mechanisms induces an increase in computational time required to simulate a given event, a more serious problem is often the severe stiffness associated with the differential equations which model the chemistry. In general, the time scales of reaction are often widely disparate. This leads to computations which routinely take hundreds of hours on supercomputers; such efforts are often impractical. Consequently, it becomes necessary to implement some strategy to reduce the stiffness introduced by the chemistry. The simplest, full equilibrium, is effectively an ILDM of dimension zero; however, this approach will necessarily miss the coupling of events which occur at time scales of chemical reaction. Simple and often useful strategies which capture some of the kinetic time scales employ explicit one-step models, e.g. Westbrook and Dryer ${ }^{1}$, or Khokhlov, et al. ${ }^{2}$. Also useful are the commonly employed partial equilibrium and steady state modeling assumptions.

As shown by Maas and Pope ${ }^{3}$, such assumptions are often not robust. While they may be useful in the range in which they have been calibrated, it is easy to find scenarios where such models cannot accurately reproduce the results of full kinetic models. As a consequence, Maas and $\mathrm{Pope}^{3}$ and simultaneously Goussis and $\mathrm{Lam}^{4}$ have advocated methods which systematically reduce chemical kinetic models in such a way that consistency with full model equations is maintained to a user-specified precision. A number of studies have appeared in recent years advancing the 
technique and some variants, $c f$. Blansenbrey, et $a l .^{5}$; Eggels, et $a l .{ }^{6} ;$ Hamiroune, et al. ${ }^{7} ; \mathrm{Lam}^{8} ;$ Schmidt, et $a l .{ }^{9}$; Norris ${ }^{10}$; and Yang and Pope ${ }^{11,12}$. In this study we have used the method of Maas and Pope, which systematically equilibrates the fast time scales and resolves the slow time scales of the reaction mechanism, thereby eliminating the associated stiffness.

Traditionally convection and diffusion in reactive flow problems are modeled by finite differences or finite elements, methods which have difficulty modeling phenomena which have localization in physical and spectral space. Here we use a WAMR, which is better suited for problems with physical and spectral localization. This technique, developed by Vasilyev and Paolucci ${ }^{13,14}$, projects the representation of the system onto a basis of wavelets. This basis has been shown to be a very efficient way to represent systems with detailed spatial structures. The capturing of the details of the structure with a small number of basis functions dramatically reduces the number of equations which need to be solved, consequently reducing computational time.

The ignition delay problem we consider is the viscous analog of the inviscid problem considered by Fedkiw, et $a .^{15}$ and is as follows. As an initial condition, a shock is taken to be propagating to the right in a mixture of hydrogen, oxygen, and argon. The shock is of insufficient strength to induce significant reaction. After some time, the shock reflects from a wall at the right, inducing a reflected, leftpropagating shock. This shock leaves the fluid adjacent to the wall in state of near zero velocity and a temperature which is sufficiently elevated to induce significant chemical reaction following a short ignition delay time.

A Strang ${ }^{16}$ operator splitting technique is used in the numerical simulation of the governing equations. This technique allows straightforward implementation of both ILDM and WAMR techniques. After initialization, the Strang-splitting progresses in a series of two-step processes. In the first step, convection and diffusion is suppressed. In this step, each point in space is treated as a well stirred reactor under constant density adiabatic conditions, and the associated ordinary differential equations are solved using the ILDM method. In the second step, the reaction source terms are deactivated, the solution at each spatial point evolves due to convection and diffusion, and the associated partial differential equations are solved using the WAMR method.

While diffusion is typically not modeled in detonation studies, it is considered here for two reasons. First, as discussed in detail by Singh, et al. ${ }^{17}$, physical diffusion is necessary to regularize predictions of flow variables downstream of the lead shock in simulations of multidimensional cellular instabilities. Whether or not this physically based regularization is crucial in determining wall tracings is an open question. Second, as will be shown here, the use of resolved physical diffusion layers correctly captures entropy layers. As discussed by Menikoff ${ }^{18}$, inviscid models using typical grid resolutions will introduce artificial entropy layers due to numerical diffusion. The coarser the grid, the more entropy introduced, and the more likely such a layer could falsely trigger temperaturesensitive chemical reaction.

The paper is organized as follows. The governing equations, initial and boundary conditions are first described. Next a detailed description of the ILDM method is given, discussing ILDM for well stirred systems and its implementation via operator splitting into systems with convection and diffusion. The WAMR technique is briefly reviewed. Detailed results are given for the shock tube test problem, and conclusions are presented.

\section{Governing Equations, Initial and Boundary Conditions}

The following equations describe the system we consider, a one-dimensional viscous mixture of $N$ ideal gases which undergo $J$ reactions.

$$
\begin{aligned}
& \frac{\partial \rho}{\partial t}+\frac{\partial}{\partial x}(\rho u)=0 \\
& \frac{\partial}{\partial t}(\rho u)+\frac{\partial}{\partial x}\left(\rho u^{2}+P-\tau\right)=0, \\
& \frac{\partial}{\partial t}\left(\rho\left(e+\frac{u^{2}}{2}\right)\right) \\
& +\frac{\partial}{\partial x}\left(\rho u\left(e+\frac{u^{2}}{2}\right)+u(P-\tau)+q\right)=0, \\
& \frac{\partial}{\partial t}\left(\rho Y_{i}\right)+\frac{\partial}{\partial x}\left(\rho u Y_{i}+j_{i}\right)=\dot{\omega}_{i} M_{i}, \\
& \dot{\omega}_{i}=\sum_{j=1}^{J} a_{j} T^{\beta_{j}} \exp \left(\frac{-E_{j}}{\Re T}\right) \nu_{i j} \prod_{k=1}^{N}\left(\frac{\rho Y_{k}}{M_{k}}\right)^{\nu_{k j}} \\
& j_{i}=-\rho \sum_{j=1}^{N} \mathcal{D}_{i j} \frac{\partial}{\partial x}\left(\frac{Y_{j}}{M_{j}} \frac{1}{\sum_{k=1}^{N} Y_{k} / M_{k}}\right), \\
& \tau=\frac{4}{3} \mu \frac{\partial u}{\partial x}, \\
& q=-k \frac{\partial T}{\partial x}
\end{aligned}
$$




$$
\begin{gathered}
P=\rho \Re T \sum_{i=1}^{N} \frac{Y_{i}}{M_{i}}, \\
e=\sum_{i=1}^{N} Y_{i}\left(h_{i}^{o}+\int_{T_{o}}^{T} c_{p i}(\hat{T}) d \hat{T}-\frac{\Re T}{M_{i}}\right) .
\end{gathered}
$$

Equations (1-3) describe the conservation of mass, linear momentum, and energy. Equation (4) is an evolution equation for species. While there are $N$ species, only $N-1$ species equations need be considered, as an equation for global mass conservation is given by Eq. (1). Equation (5) is a constitutive equation for the evolution of species which is the law of mass action with Arrhenius kinetics. Equations (6-8) give constitutive relations for mass, momentum, and energy diffusion which are the ordinary Fick's law, Newtonian stress-strain rate relation, and Fourier's law, all with assumed constant diffusion coefficients. The contribution to the heat flux due to species diffusion has been neglected here, though this is easily remedied. Equations (9-10) are thermal and caloric state equations for a mixture of ideal gases with temperature-dependent specific heats.

The independent variables are time $t$ and position $x$. The dependent variables, $\rho, u, P, \tau, e, q, Y_{i}$, $j_{i}, \omega_{i}$, and $T$, describe the density, velocity, pressure, viscous stress, internal energy per unit mass, diffusive energy flux, mass fraction of species $i$ (with $i=1, \ldots, N)$, diffusive mass flux of species $i$, molar production rate of species $i$, and temperature, respectively. The specific heat of species $i, c_{p i}$, is taken to be a known function of temperature. The function is in the form of a standard polynomial curve fit found in the Chemkin III thermodynamic data base. ${ }^{19}$ The kinetic model, identical to that used by Fedkiw, et al. ${ }^{15}$, was originally developed by Maas and Warnatz ${ }^{20}$ and considers the reaction of $\mathrm{N}=9$ species $\left(\mathrm{H}, \mathrm{O}, \mathrm{H}_{2}\right.$, $\left.\mathrm{O}_{2}, \mathrm{OH}, \mathrm{H}_{2} \mathrm{O}, \mathrm{HO}_{2}, \mathrm{H}_{2} \mathrm{O}_{2}, \mathrm{Ar}\right)$ in $\mathrm{J}=37$ reactions. Coefficients for this mechanism are listed in Table 1.

Parameters in Eqs. (1-10) are $M_{i}, a_{j}, \beta_{j}, E_{j}, \Re, \nu_{i j}$, $\mathcal{D}_{i j}, \mu, k$, and $h_{i}^{o}$. They represent the molecular mass of species $i$, the kinetic rate constant of reaction $j$, the temperature dependency exponent of reaction $j$, the activation energy of reaction $j$, the universal gas constant $(\Re=8.31441 \mathrm{~J} / \mathrm{mol} / \mathrm{K})$, the stoichiometric coefficient of the $i$ th species of reaction $j$, the mass diffusivity of the $i$ th species due to the $j$ th species, the dynamic viscosity, the thermal conductivity, and the standard enthalpy of formation of species $i$.

For the present study, for expediency we make the simplifying assumptions that all diffusion coefficients have no dependency on species, nor do they have temperature dependency; moreover, we have chosen their values to be somewhat larger than realistic values for this system. We take $\mathcal{D}_{i j}=5.6 \times 10^{-3} \mathrm{~m}^{2} / \mathrm{s}$, $\mu=1.0 \times 10^{-3} \mathrm{~N} \cdot \mathrm{s} / \mathrm{m}^{2}$, and $k=8.3 \times 10^{0} \mathrm{~W} / \mathrm{m} / \mathrm{K}$. More realistic variable properties will be incorporated into future work.

We consider a shock tube of length $0.12 \mathrm{~m}$ filled initially with $\mathrm{H}_{2}, \mathrm{O}_{2}$, and $\mathrm{Ar}$ in a 2/1/7 molar ratio. For $0 \leq x \leq 0.06 \mathrm{~m}$, the gas is taken to be at $\rho=0.18075 \mathrm{~kg} / \mathrm{m}^{3}, u=487.34 \mathrm{~m} / \mathrm{s}$, and $P=35.594 k P a$. For $0.06<x \leq 0.12 \mathrm{~m}$, the gas is at $\rho=0.072 \mathrm{~kg} / \mathrm{m}^{3}, u=0 \mathrm{~m} / \mathrm{s}$, and $P=7.173 \mathrm{kPa}$. This state is consistent with Rankine-Hugoniot jump conditions for the inviscid equivalent of Eqs. (1-10). Knowledge of these parameters allows determination of all other dependent variables at $t=0 \mathrm{~s}$ through the use of the governing equations. These two states are linked by an inverse hyperbolic tangent function interpolation distributed over a thin spatial scale to suppress discontinuities in the initial state.

At $x=0.12 \mathrm{~m}$, we consider a boundary which is closed and adiabatic. Consequently $u=0 \mathrm{~m} / \mathrm{s}$, and additionally diffusive mass and energy fluxes $j_{i}$, $q$ must be zero. At $x=0 \mathrm{~m}$, we allow inflow conditions of $u=487.34 \mathrm{~m} / \mathrm{s}, \rho=0.18075 \mathrm{~kg} / \mathrm{m}^{3}$, $P=35.594 k P a$.

\section{ILDM for a Well Stirred Reactive System}

In the operator splitting technique which is employed to solve Eqs. (1-10), each discrete point in space is treated as an adiabatic, well stirred reactor, thus enabling the use of the ILDM technique. The method provides a systematic way to overcome the severe stiffness which is associated with full models of gas phase combustion, and thus significantly improves computational efficiency. In a well stirred calculation, the full model predicts the ratio of the time scale of the slowest reaction mode to that of the fastest reaction mode to reach values near $10^{7}$, which indicates severe stiffness is present. The ILDM method systematically eliminates most of this stiffness by equilibrating fast time scale events and describing parametrically a low dimensional manifold upon which slow time scale events evolve.

Here, the method of Maas and Pope ${ }^{3}$ is summarized. The constant density, isochoric, adiabatic combustion of a well-stirred system of $N$ species constituted from $L$ elements can be expressed as $N$ nonlinear ordinary differential equations, which evolve in an $N$-dimensional composition (phase) space:

$$
\frac{d Y_{i}}{d t}=\frac{\dot{\omega}_{i} M_{i}}{\rho_{o}}, \quad i=1, \ldots, N,
$$

where $\rho_{o}$ is the constant density of the mixture. While mass conservation could be used to reduce the 
dimension of this system by one, at this point we choose not to, for reasons which will become obvious.

It is easily shown that for a constant density, isochoric, adiabatic system, the mass-averaged specific internal energy remains constant. Further, if the gases in the mixture are thermally perfect, the specific internal energy of each component is at most a function of temperature. Hence, knowledge of the mass fractions and mass-averaged specific internal energy allows one to solve for the temperature $T$ in terms of those variables by solving the following nonlinear algebraic equation via Newton's method

$$
e_{o}=\sum_{i=1}^{N} e_{i}(T) Y_{i}
$$

where $e_{o}$ is the constant internal energy of the mixture and $e_{i}$ is the internal energy of the $i$ th species at temperature $T$. Hence, $\dot{\omega}_{i}$, which is in general a function of temperature, density, and mass fractions, can now be considered as a function only of mass fractions for fixed values of $e_{o}$ and $\rho_{o}$ :

$$
\begin{aligned}
\dot{\omega}_{i} & =\dot{\omega}_{i}\left(T\left(e_{o}, Y_{1}, \ldots, Y_{N}\right), Y_{1}, \ldots, Y_{N} ; \rho_{o}\right), \\
& =\dot{\omega}_{i}\left(Y_{1}, \ldots, Y_{N} ; e_{o}, \rho_{o}\right) .
\end{aligned}
$$

Both $\dot{\omega}_{i}$ and $e_{i}(T)$ can be easily evaluated using the Chemkin III $^{21}$ package.

At fixed $e_{o}$ and $\rho_{o}$, Eq. (11) can be re-written as

$$
\frac{d \mathbf{Y}}{d t}=\mathbf{F}(\mathbf{Y})
$$

where $\mathbf{Y}=\left(Y_{1}, \ldots, Y_{N}\right)^{T}$ and $\mathbf{F}(\mathbf{Y})=$ $\left(\frac{\dot{\omega}_{1} M_{1}}{\rho_{o}}, \ldots, \frac{\dot{\omega}_{N} M_{N}}{\rho_{o}}\right)$. The reciprocals of the eigenvalues of the Jacobian $\mathbf{F}_{\mathbf{Y}}$ at any point in the composition space identify the $N$ associated characteristic time scales. The corresponding eigenvectors identify the directions in which each eigenmode of the total trajectory evolves in composition space. The ILDM is identified as a set of points in the composition space where the composition space velocity vector $\mathbf{F}$ is orthogonal to the eigenvectors associated with fast time scales. Orthogonal real Schur vectors are used as the basis for the fast and slow time scales instead of the eigenvectors, which may be difficult to evaluate. These difficulties are associated with the Jacobian $\mathbf{F}_{\mathbf{Y}}$ having zero eigenvalues, nearly equal eigenvalues, or complex eigenvalues. The Jacobian matrix has $L$ zero eigenvalues associated with the conservation of $L$ atomic elements, which is equivalent to enforcing mass conservation.

We then choose to resolve $M$ slow time scales; this will result in the choice of $M$ species which will form part of the parameterization for the complete ILDM.
An ILDM associated with the $M$ slowest time scales is given by

$$
\mathbf{Q}_{L} \cdot \mathbf{F}(\mathbf{Y})=0
$$

where

$$
\mathbf{Q}_{L}=\left(\begin{array}{ccc}
- & q_{L+M+1}^{T} & - \\
- & q_{L+M+2}^{T} & - \\
& \vdots & \\
- & q_{N}^{T} & -
\end{array}\right)
$$

Here $\mathbf{Q}_{L}$ is defined using the following real Schur decomposition of the Jacobian matrix

$$
\begin{gathered}
\mathbf{F}_{\mathbf{Y}}=\mathbf{Q} \cdot \mathbf{N} \cdot \mathbf{Q}^{T} \\
\mathbf{Q}=\left(\begin{array}{ccc}
\mid & & \mid \\
q_{1} & \cdots & q_{N} \\
\mid & & \mid
\end{array}\right), \mathbf{Q}^{T}=\left(\begin{array}{ccc}
- & q_{1}^{T} & - \\
& \vdots & \\
- & q_{N}^{T} & -
\end{array}\right)
\end{gathered}
$$

where $\mathbf{Q}$ is the matrix of real Schur vectors and $\mathbf{N}$ is an upper-triangular matrix with eigenvalues along the main diagonal in decreasing order. The matrix $\mathbf{Q}_{L}$ is composed of row vectors defining the subspace associated with the $N-(L+M)$ fastest time scales. For a proof of the above formulation, see Ref. 3 . Thus, for fixed energy, density, and element concentrations, the ILDM method identifies $M$-dimensional subspaces $(M<N-L)$ on which slower time scale events evolve. For effectively one-step chemistry, we take $M=1$.

To construct the manifold, one first determines the equilibrium point of the system and the local real Schur decomposition, which gives local eigenvalues and associated orthonormal Schur vectors for a system linearized about the equilibrium point. One then perturbs $M$ of the species away from equilibrium to prescribed values to form $M$ algebraic equations

$$
Y_{i}=Y_{i_{o}} \quad \text { for } i=1, \ldots, M .
$$

These are solved simultaneously with $N-(L+M)$ algebraic ILDM equations, (15), and $L$ linear equations associated with element conservation

$$
\sum_{i=1}^{N} \phi_{j, i} \frac{Y_{i}}{M_{i}}=\frac{\Xi_{j o}}{M_{j}} \quad \text { for } \quad j=1, \ldots, L,
$$

where $\phi_{j, i}$ are the number of moles of element $j$ in species $i$. Here, $\Xi_{j_{o}}$ is the mass fraction of the $j$ th element and is constant for a given calculation. However, variable element concentrations must be considered for problems with preferential diffusion or with non-uniform initial concentrations, so in general, the manifold must be parameterized by $\Xi_{j o}$. 
These equations are solved with a predictor corrector technique coupled with a Newton's method iteration to solve for the mass fractions at a new point on the manifold. The new manifold point is used as a seed for calculation of further points. An arclength parameterization of the manifold is employed to overcome difficulties associated with turning points of the manifold in composition space. This method can also be extended for computing manifolds for an adiabatic, isobaric system.

With this analysis, an $M$-dimensional manifold can be identified in an $N$-dimensional composition space for a given set of $\rho_{o}, e_{o}, \Xi_{1_{o}}, \ldots, \Xi_{L_{o}}$. These are the ILDMs that are traditionally discussed in the literature. Thus, a different ILDM is required for a different set of densities, internal energies and element mass fractions. Since in general calculations, one can expect all of these quantities to vary, the actual relevant manifold which must be formed has dimension $K=M+2+L$ and can be tabulated numerically to give

$$
\begin{aligned}
Y_{i} & =Y_{i}\left(Y_{1}, \ldots, Y_{M}, e, \rho, \Xi_{1}, \ldots, \Xi_{L}\right), \\
\text { for } i & =M+1, \ldots, N .
\end{aligned}
$$

where $Y_{1}, \ldots, Y_{M}$ are the chosen reference variables for the ILDM lookup table. The reference variables are chosen in such a way that the ILDM is single valued with respect to these variables for easy lookup. While there is no guarantee of single-valuedness, in the problems studied, we have found it to be the case.

It is the dimension of the lookup table, $K$, which is critically important in manifold methods. Many previously reported calculations have been restricted to premixed conditions in the isobaric and/or adiabatic limits, thus reducing the dimension. In the calculations presented here, variable energy and density are intrinsic features of the flow; given that we have chosen $M=1$ and for the hydrogen, oxygen, argon system, $L=3$, we in principle must deal with a table which has dimension $K=6$. Because we study a uniformly premixed mixture, and because we have ignored differential diffusion effects, the element concentrations remain constant throughout the calculations, reducing the effective dimension of the manifold here to three; the variables of manifold parameterization are chosen to be $e, \rho$, and $Y_{\mathrm{H}_{2} \mathrm{O}}$.

With $M=1$, a projection of the ILDM for fixed internal energy, density, and element concentration for the hydrogen/oxygen/argon system is plotted in Fig. 1 with $Y_{\mathrm{H}_{2} \mathrm{O}}$ used as the reference independent variable for the ILDM and $Y_{\mathrm{H}_{2} \mathrm{O}_{2}}$ as the dependent variable. Mass fractions for all species, not shown

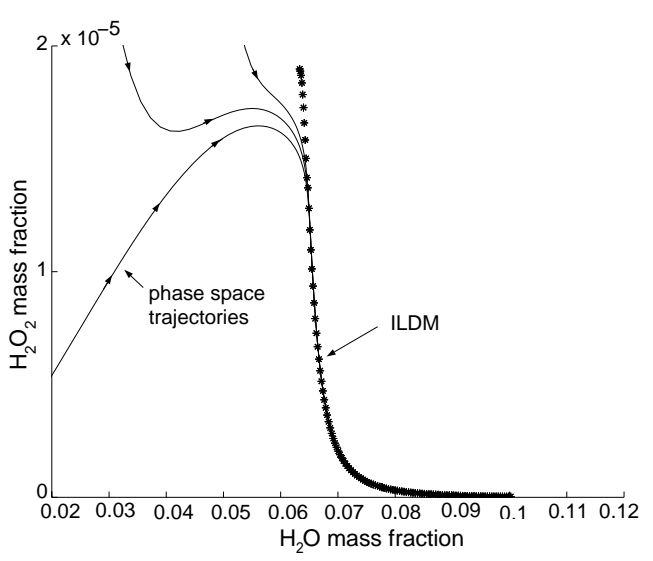

Figure 1: ILDM for 9 species 37 step reaction mechanism of hydrogen/oxygen/argon combustion as function of $Y_{\mathrm{H}_{2} \mathrm{O}}$ at constant density, energy, and element concentrations, along with trajectories from full time integration showing relaxation to the manifold.

here, are also available as functions of $Y_{\mathrm{H}_{2} \mathrm{O}}$. Also shown on the plot are projections of trajectories in this two-dimensional subspace for a variety of initial conditions. It is seen that all trajectories relax to the ILDM. Though not obvious from Fig. 1, the relaxation from the initial state to the manifold occurs on a relatively fast time scale, while once on the ILDM, the subsequent relaxation to final equilibrium occurs on a much slower time scale.

A projection of the $M=1$ ILDM for varying internal energy, constant density, and constant element concentrations for the hydrogen/oxygen/argon system is plotted in Fig. 2, again with $Y_{\mathrm{H}_{2} \mathrm{O}}$ used as the reference independent variable and $\mathrm{Y}_{\mathrm{H}_{2} \mathrm{O}_{2}}$ as the dependent variable. Here the energy levels were chosen to be consistent with those realized in the detonation calculation.

\section{Operator Splitting}

The governing equations (1-10) can be written in the following compact form

$$
\frac{\partial \mathbf{x}}{\partial t}=\mathbf{F}(\mathbf{x})-\mathbf{\Pi}(\mathbf{x})
$$

where $\mathbf{x}$, representing a new set of conserved dependent variables, $\mathbf{F}(\mathbf{x})$, representing a reaction source term, and $\mathbf{\Pi}(\mathbf{x})$, representing convection and diffu- 


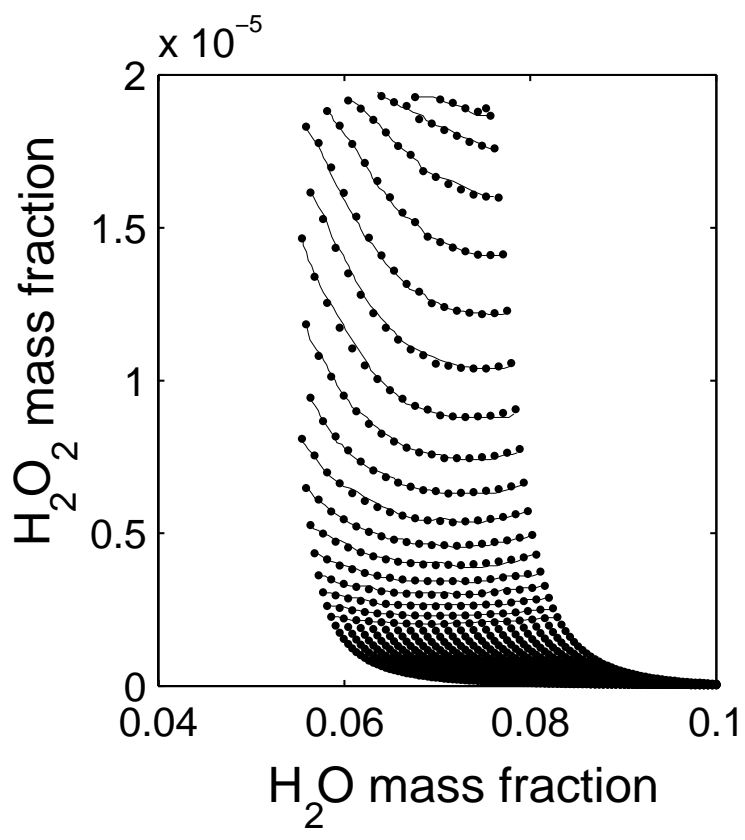

Figure 2: ILDM for 9 species 37 step mechanism of hydrogen oxygen argon combustion as function of $Y_{\mathrm{H}_{2} \mathrm{O}}$ at constant density, constant element concentration, and variable energy. Dots indicate actual points which are tabulated.

sion, are given by

$$
\begin{gathered}
\mathbf{x}=\left(\begin{array}{c}
\rho \\
\rho u \\
\rho\left(e+\frac{u^{2}}{2}\right) \\
\rho Y_{1} \\
\vdots \\
\rho Y_{N-1}
\end{array}\right), \quad \mathbf{F}(\mathbf{x})=\left(\begin{array}{c}
0 \\
0 \\
0 \\
\dot{\omega}_{1} M_{1} \\
\vdots \\
\dot{\omega}_{N-1} M_{N-1}
\end{array}\right) \\
\mathbf{\Pi}(\mathbf{x})=\left(\begin{array}{c}
\frac{\partial}{\partial x}(\rho u) \\
\frac{\partial}{\partial x}\left(\rho u^{2}+P-\tau\right) \\
\left.\rho u\left(e+\frac{u^{2}}{2}\right)+u(P-\tau)+q\right) \\
\frac{\partial}{\partial x}\left(\rho u Y_{1} j_{1}\right) \\
\vdots \\
\frac{\partial}{\partial x}\left(\rho u Y_{N-1} j_{N-1}\right)
\end{array}\right)
\end{gathered}
$$

The forms shown above for $\mathbf{F}$ and $\boldsymbol{\Pi}$ are actually given in terms of primitive dependent variables; through lengthy algebraic manipulation, omitted here, it is possible to cast all primitive dependent variables in terms of the new dependent variables $\mathbf{x}$. Equation (22) is solved in two steps using Strangsplitting ${ }^{16}$
In step 1, the following equation, equivalent to that for a well stirred reactor at every point in the space, is solved

$$
\frac{\partial \mathbf{x}}{\partial t}=\mathbf{F}(\mathbf{x}) .
$$

The first three equations of Eq. (24) are homogeneous and can be integrated exactly to give

$$
\rho=\rho_{o}, \quad u=u_{o}, \quad e=e_{o}
$$

Hence, the species equations in Eq. (24) reduce to the following ordinary differential equations which are solved at each point in space with $\rho, u$ and $e$ held constant every time Step 1 is repeated.

$$
\frac{d Y_{i}}{d t}=\frac{\dot{\omega}_{i} M_{i}}{\rho_{o}}, \quad i=1, \ldots, N-1 .
$$

Eq. (26) is essentially the same as Eq. (11) for the well stirred reactor. Since $\rho_{o}$ is constant, $Y_{N}$ can be determined algebraically. Equation (26) can be solved using the ILDM method if the composition of the mixture at spatial point $k$ is close to the ILDM. When using ILDMs with $M=1$, we need to integrate only one ordinary differential equation associated with the reference variable, which is chosen to be $Y_{\mathrm{H}_{2} \mathrm{O}}$. For the given $\rho_{o}, u_{o}$, and $e_{o}$, the rest of the mass fractions and temperature are evaluated using the ILDM lookup tables.

If the composition is far from the ILDM, it is important to use integration of the full reaction kinetics equations; we achieve this using the software package $\mathrm{LSODE}^{22}$ in implicit mode. While this reduces the efficacy of the ILDM method, it is critical to avoid large phase errors associated with projecting onto the manifold from a remote region of phase space. In other words, while all processes are typically destined to reach the manifold, it is critical for the proper sequencing of events that they reach the manifold at the correct time, and reach the right point on the manifold. A naive projection can easily give plausible answers which unfortunately have $O(1)$ errors associated with them. In practice, in our calculations, we find we are able to use the ILDM method in cells which have recently been shocked, and thus had their reactions activated.

In step 2, the following equation, which is a set of partial differential equations, is solved for the convection diffusion step:

$$
\frac{\partial \mathbf{x}}{\partial t}=-\mathbf{\Pi}(\mathbf{x}) .
$$

The convection-diffusion step is equivalent to a perturbation off the ILDM. Subsequent to the perturbation, there is a fast relaxation to a new manifold 
corresponding to a new set of conserved variables. This is accomplished by orthogonal projection onto the new manifold. Orthogonal projection is allowed by the large time scale difference between slow chemical/fluid time scales and fast chemical time scales as long as convective and diffusive effects are not large. Figure 3 depicts how this projection is accomplished. In contrast to many implementations of the ILDM

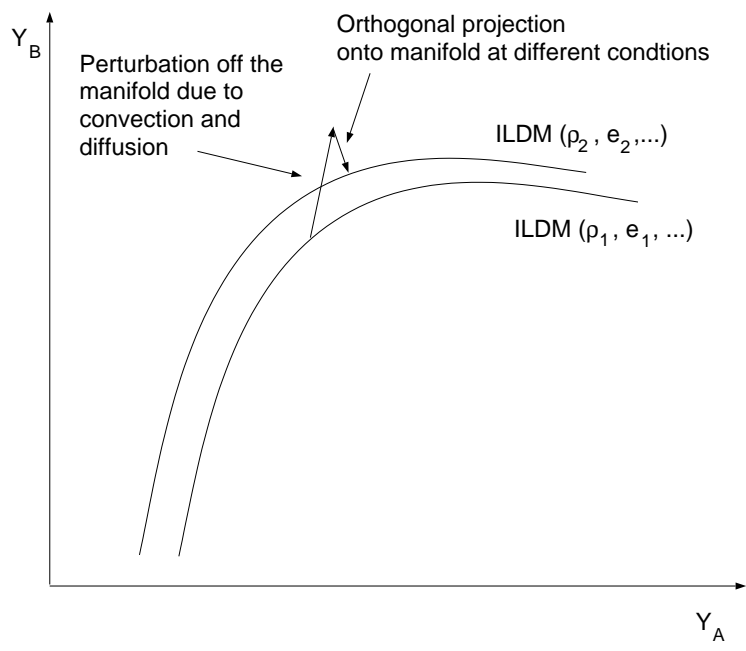

Figure 3: Sketch of projection to manifold of new energy and density levels following perturbation due to convection and diffusion.

method for partial differential equations, we convect and diffuse all variables, not just slow variables associated with the ILDM. While this comes at a cost of solving more equations, it is absolutely necessary to preserve the consistency of the Strang operator splitting method.

\section{$\underline{\text { Wavelets }}$}

The WAMR technique, discussed in detail by Vasilyev and Paolucci ${ }^{13,14}$, is summarized here. At any given time step, the pressure, temperature, density, and velocity fields are projected onto a multilevel wavelet basis. The amplitudes of the wavelet basis functions give a measure of the importance of a particular wavelet mode. Additionally, one has available a priori error estimates, in contrast to most gradient-based adaptive mesh refinement techniques. All wavelets whose amplitude are below a defined threshold are removed. Calculations are performed for each wavelet whose amplitude is above the threshold (essential wavelets) and for a certain number below the threshold (neighboring wavelets). If at the completion of a time step, an essential wavelet has its amplitude drop below the threshold, it is reclassified as a neighboring wavelet or eliminated, and the neighboring region is adjusted; similarly, if a wavelet in the neighboring region has its amplitude become sufficiently large, it is reclassified as an essential wavelet, and the neighboring region is adjusted.

The method is based on a collocation strategy using the auto-correlation function of the Daubechies scaling function of order five as the basis. A simple linearized trapezoidal method is used for time advancement. Because an implicit method is used, there is a much looser time step restriction than for explicit methods. It is found that the key factor which limits the time step is the size of the neighboring region, which restricts how far a wave can propagate in a given time step.

\section{$\underline{\text { Results }}$}

Results for the shock tube calculations are given here. Figure 4 gives predictions of temperature, velocity, pressure, and density vs. distance at time $t=195 \mu \mathrm{s}$. At this time, the lead right-traveling
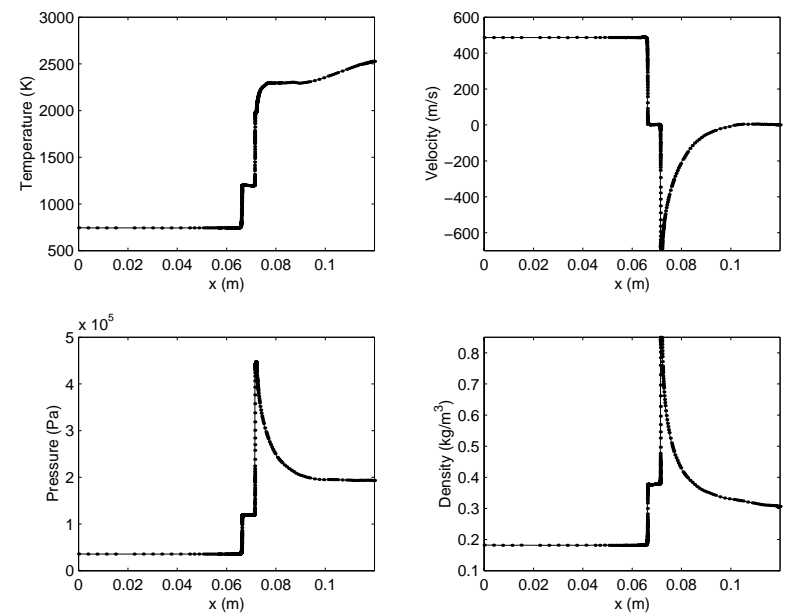

Figure 4: Predictions of temperature, velocity, pressure, and density vs. distance at $t=195 \mu \mathrm{s}$ using a maximum of 300 collocation points, 15 scale levels for full chemical kinetics (solid lines) and ILDM kinetics (dots) for viscous hydrogen/oxygen detonation.

inert shock has reflected off the right wall and is propagating to the left with its head near $x=0.065 \mathrm{~m}$. Close behind the lead shock is the much stronger, left-propagating ZND detonation wave, with its head near $x=0.072 \mathrm{~m}$. All of the usual salient features of a ZND detonation are predicted here. The von Neumann spike is predicted at a pressure of around $450 \mathrm{kPa}$, and the pressure relaxes to near $200 \mathrm{kPa}$ 
at the right boundary. The post-detonation temperatures are near $2,500 K$, and the velocity is seen to relax to a value of zero at the right boundary.

The solid lines show the predictions of the full chemical kinetics model. The dots show the results of the calculations using the ILDM resolving one reaction time scale; this can be interpreted as one-step chemistry with a rational fidelity to full chemical kinetics. It is seen on this scale that the predictions are nearly identical. Examination of the local eigenvalues indicates that use of the manifold suppresses temporal resolution of reaction events which occur faster than a roughly $0.1 \mu s$ time scale. We have not yet performed detailed studies of how much the ILDM method improves the overall computational efficiency for this problem; on simpler test problems, we have predicted an increase of roughly a factor of ten. The calculation itself took roughly ten hours on a $330 \mathrm{MHz}$ Sun Ultra10 workstation.

Figure 5 shows similar results for the species mass fractions at the same time, $t=195 \mu s$. Steep gra-
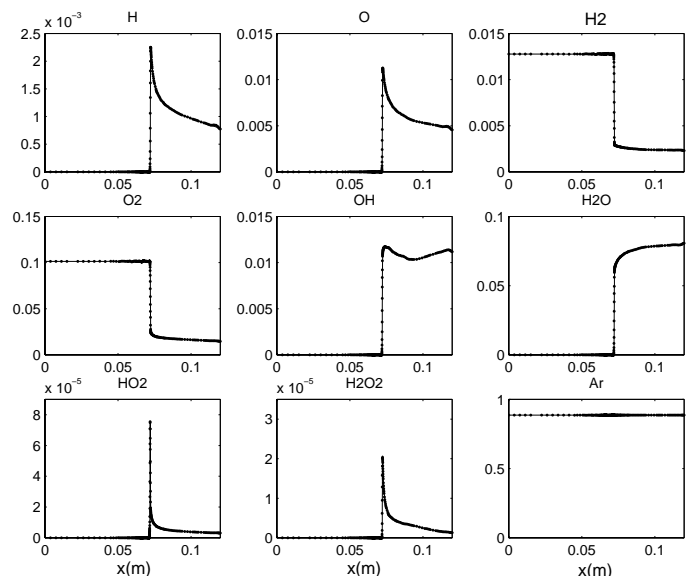

Figure 5: Predictions of species mass fractions vs. distance at $t=195 \mu \mathrm{s}$ using a maximum of 300 collocation points, 15 scale levels for full chemical kinetics (solid lines) and ILDM kinetics (dots) for viscous hydrogen/oxygen detonation.

dients in mass fractions are predicted near the detonation front. As expected, $\mathrm{HO}_{2}, \mathrm{H}$, and $\mathrm{H}_{2} \mathrm{O}_{2}$ mass fractions have relatively small values which peak at the detonation front. Under these conditions, the major product is $\mathrm{H}_{2} \mathrm{O}$. On the length scales shown in Figs. 4 and 5, the results appear very similar to the inviscid predictions of Fedkiw, et al. ${ }^{15}$

Figure 6 demonstrates the adaptive nature of the WAMR. It shows the distribution of collocation
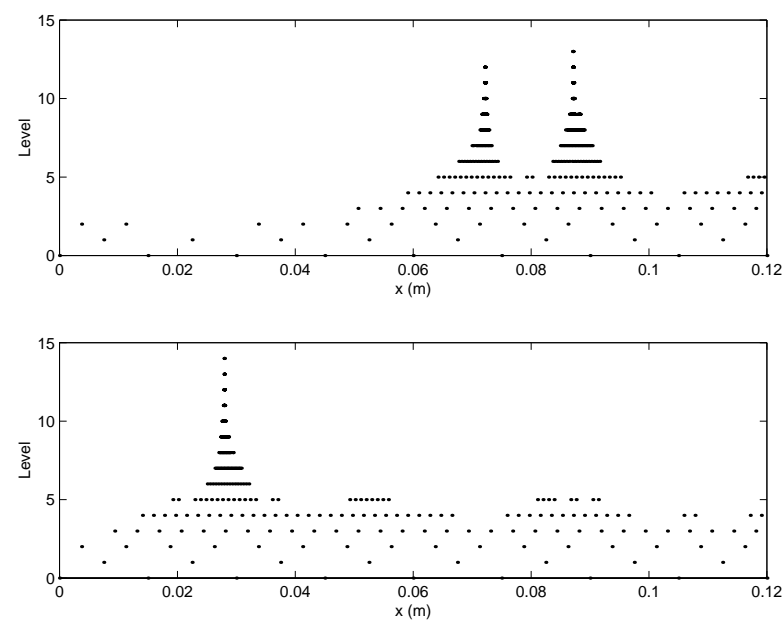

Figure 6: Spatial distribution of collocation points and levels at $t=180 \mu \mathrm{s}$ (two shock structure) $t=$ $230 \mu s$ (single shock structure) demonstrating grid adaptation.

points and their scale levels, $2^{-j}, j=0, \ldots, 14$, at two different times, first at $t=180 \mu \mathrm{s}$, when the lead shock and the approaching detonation are present, and later at $t=230 \mu \mathrm{s}$, after they have merged. In both cases, at most three hundred collocation points and fifteen wavelet scale levels were sufficient to capture the flow features to the prescribed dimensionless error tolerance of $10^{-4}$. Moreover, it is clear that the algorithm adapts to the features of the flow.

The effects of diffusion are clearly seen when one examines finer length scales. Figure 7 shows two views of pressure vs. distance at a somewhat later time, $t=230 \mu \mathrm{s}$, by which time the detonation wave has overtaken the reflected shock. In the view on the
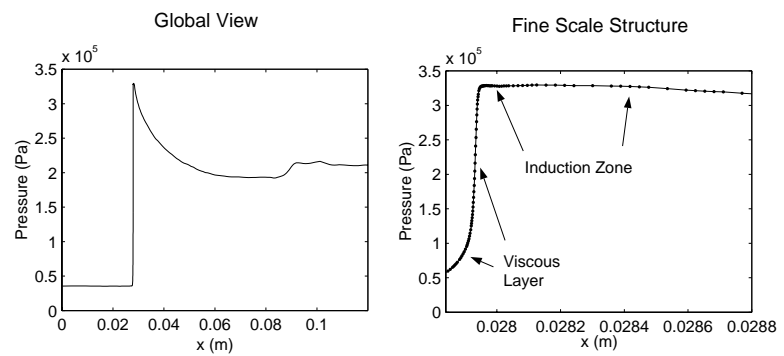

Figure 7: Predictions of pressure vs. distance at $t=230 \mu s$ on a coarse and fine length scales demonstrating the spatial resolution of viscous and induction zone structures.

left, the same length scale is shown as in Fig. 4. The view on the right shows a 120 factor spatial magnifi- 
cation near the lead shock. In this figure the dots represent the actual collocation points as chosen by the WAMR technique. It is clear on this scale that both the viscous shock and chemical induction zone have been resolved. Here it is predicted that the shock is essentially inert and has a thickness of roughly $50 \mu \mathrm{m}$. Lastly, while the validity of the continuum assumption is often questioned for viscous shock calculations, Liepmann and Roshko ${ }^{23}$ show that for a sufficiently weak shocks, predictions of shock thicknesses from continuum models agree surprisingly well with experiment and theories that account for sub-continuum effects. Their results are for a shock traveling at Mach number 1.82 in helium.

The induction zone, a region of essentially constant pressure, temperature, and density, has a thickness of roughly $470 \mu \mathrm{m}$. In the induction zone many reactions are occurring, giving rise to a release of energy which, because of the extreme temperature sensitivity of reaction rates, accumulates to an extent that a thermal explosion occurs at the end of the induction zone. While the WAMR certainly has captured these thin layers, it is noted that because it was chosen not to use individual species mass fractions as part of the adaptation criteria, that some finer scale reaction zone structures have not been spatially resolved.

As discussed by Menikoff ${ }^{18}$, inviscid codes introduce pseudo-entropy layers near regions of wave-wave and wave-boundary interactions. These often appear as $O(1)$ anomalies in temperature and density near the wall in shock tube predictions. Figure 8 shows the results of our viscous calculation in a spatial zone near the wall just after shock reflection. On this scale, there is no apparent entropy layer near the wall.

A finer scale examination of the dependent variables, shown in Figure 9, reveals what is happening. It is evident from the temperature plot that there is a small entropy layer near the wall, here physically induced. The physical diffusion mechanisms rapidly smears the layer within a few microseconds. It may be possible that the correct capturing of a temperature-sensitive ignition event near a wall could be critically dependent on having the correct physics in the model. For the viscous calculation, a temperature rise of roughly $5 K$ is predicted. Performing a similar calculation with an inviscid model using 400 evenly spaced grid points induces a temperature rise of nearly $20 \mathrm{~K}$.

\section{$\underline{\text { Conclusions }}$}

The ILDM technique offers an effective way to rationally reduce detailed kinetics models; here, an effectively one step model was used. The advantage
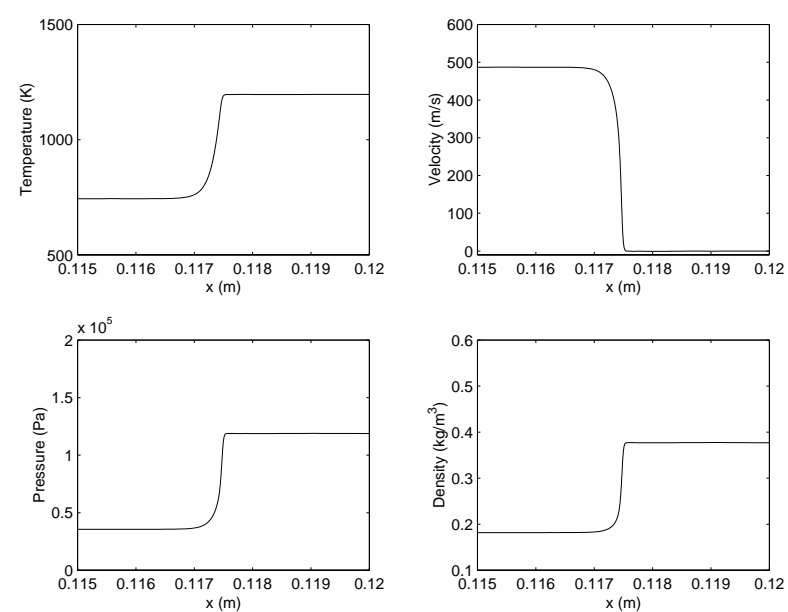

Figure 8: Predictions of temperature, velocity, pressure, and density vs. distance before commencement of significant reaction but after shock reflection using a reactive Navier-Stokes model.

is that the reduced model is guaranteed to maintain fidelity to full kinetic models to within a time scale which is easily determined. The WAMR technique allows the attainment of dramatic spatial resolution for flows, such as viscous hydrogen/oxygen detonations, with widely disparate spatial scales. Here, thin viscous shocks, entropy layers, and induction zones were fully resolved along with phenomena which evolved on much larger laboratory scales. While there are many challenges remaining for both methods, including how to efficiently build the higher dimensional ILDMs which will be necessary to resolve finer time scales, and extending the WAMR technique to complex multidimensional geometry, it appears clear that these methods can be used effectively to solve problems with disparate scales which are endemic in scientific computing.

\section{References}

${ }^{1}$ Westbrook, C. K., and Dryer, F. L., "Simplified Reaction Mechanisms for the Oxidation of Hydrocarbon Fuels in Flames," Combustion Science and Technology, Vol. 27, 1981, pp. 31-43.

${ }^{2}$ Khokhlov, A. M., Oran, E. S., Chtchelkanova, A. Y., and Wheeler, J. C., "Interaction of a Shock with a Sinusoidally Perturbed Flame," Combustion and Flame, Vol. 117, Nos. 1-2, 1999, pp. 99-116.

${ }^{3}$ Maas, U., and Pope, S. B., "Simplifying Chemical Kinetics: Intrinsic Low-Dimensional Manifolds in Composition Space," Combustion and Flame, Vol. 88, Nos. 3-4, 1992, pp. 239-264. 

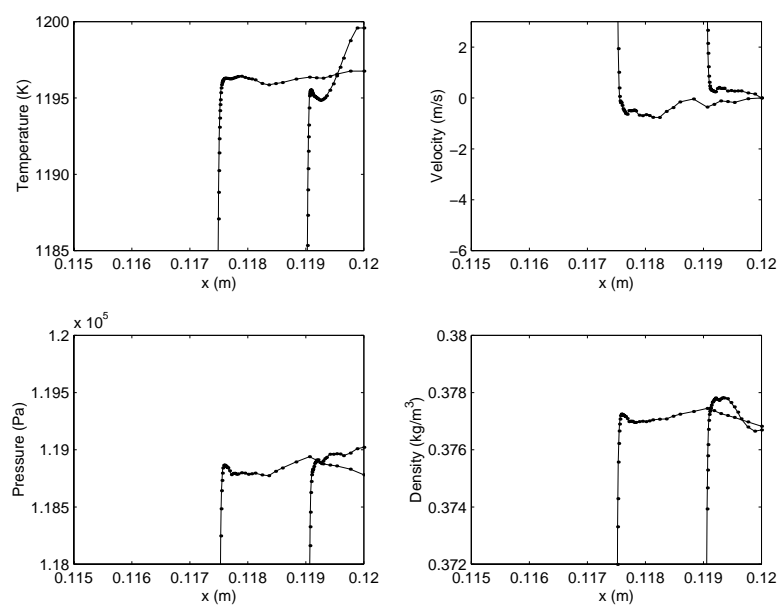

Figure 9: Close-up view of predictions of temperature, velocity, pressure, and density vs. distance before commencement of significant reaction but just after shock reflection.

${ }^{4}$ Goussis, D. A., and Lam, S. H., "A Study of Homogeneous Methanol Oxidation Kinetics Using CSP," Twenty-Fourth Symposium (International) on Combustion, The Combustion Institute, 1992, pp. 113-120.

${ }^{5}$ Blasenbrey, T., Schmidt, D., and Maas, U., "Automatically Simplified Chemical Kinetics and Molecular Transport and Its Applications in Premixed and Non-Premixed Laminar Flame Calculations," Twenty-Seventh International Symposium on Combustion, The Combustion Institute, 1998.

${ }^{6}$ Eggels, R. L. G. M., Louis, J. J. J, Kok, J. B. W., and DeGoey, L. P. H., "Comparison of Conventional and Low-Dimensional Manifold Methods to Reduce Reaction Mechanisms," Combustion Science and Technology, Vol. 123, Nos. 1-6, 1997, pp. 347-362.

${ }^{7}$ Hamiroune, D., Bishnu, P., Metghalchi, M., and Keck, J. C., "Rate-Controlled ConstrainedEquilibrium Method Using Constraint Potentials," Combustion Theory and Modeling, Vol. 2, No. 1, 1998, pp. 81-94.

${ }^{8}$ Lam, S. H., "Using CSP to Understand Complex Chemical Kinetics," Combustion Science and Technology, Vol. 89, Nos. 5-6, 1993, pp. 375-404.

${ }^{9}$ Schmidt, D., Segatz, J., Riedel, U., Warnatz, J., and Maas, U., "Simulation of Laminar Methane-Air Flames using Automatically Simplified Chemical Kinetics," Combustion Science and Technology, Vols. 113-114, 1996, pp. 3-16.

${ }^{10}$ Norris, A. T., "Automated Simplification of Full Chemical Mechanisms: Implementation in National Combustion Code," AIAA Paper 98-3987, July 1998.
${ }^{11}$ Yang, B., and Pope, S. B., "An Investigation of the Accuracy of Manifold Methods and Splitting Schemes in the Computational Implementation of Combustion Chemistry," Combustion and Flame, Vol. 112, Nos. 1-2, 1998, pp. 16-32.

${ }^{12}$ Yang, B., and Pope, S. B., "Treating Chemistry in Combustion with Detailed Mechanisms-In Situ Adaptive Tabulation in Principal DirectionsPremixed Combustion," Combustion and Flame, Vol. 112, Nos. 1-2, 1998, pp. 85-112.

${ }^{13}$ Vasilyev, O. V., and Paolucci, S., "A Dynamically Adaptive Multilevel Wavelet Collocation Method," Journal of Computational Physics, Vol. 125, No. 2, 1996, pp. 498-512.

${ }^{14}$ Vasilyev, O. V., and Paolucci, S., "A Fast Adaptive Wavelet Collocation Algorithm for Multidimensional PDEs," Journal of Computational Physics, Vol. 138, No. 1, 1997, pp. 16-56.

${ }^{15}$ Fedkiw, R. P., Merriman, B., and Osher, S., "High Accuracy Numerical Methods for Thermally Perfect Gas Flows with Chemistry," Journal of Computational Physics, Vol. 132, No. 2, 1997, pp. 175-190.

${ }^{16}$ Strang, G., "On the Construction and Comparison of Difference Schemes," SIAM Journal on $\mathrm{Nu}$ merical Analysis, Vol. 5, 1968, pp. 506-517.

${ }^{17}$ Singh, S., Powers, J. M., and Paolucci, S., "Detonation Solutions from Reactive Navier-Stokes Equations," AIAA Paper 99-0966, Jan. 1999.

${ }^{18}$ Menikoff, R., "Errors when Shock-Waves Interact Due to Numerical Shock Width," SIAM Journal on Scientific Computing, Vol. 15, No. 5, 1994, pp. 1227-1242.

${ }^{19}$ Kee, R. J., Rupley, F. M., and Miller, J. A., "The Chemkin Thermodynamic Data Base," Sandia National Laboratories Report SAND87-8215B, 1990.

${ }^{20}$ Maas, U., and Warnatz, J., "Ignition Processes in Hydrogen-Oxygen Mixtures," Combustion and Flame, Vol. 74, No. 1, 1988, pp. 53-69.

${ }^{21}$ Chemkin-III, A Software Package for the Analysis of Gas-Phase Chemical and Plasma Kinetics, Reaction Design, CHE-035-1, 1999.

${ }^{22}$ Hindmarsh, A. C., "ODEPACK, A Systematized Collection of ODE solvers," in Scientific Computing, R. S. Stepleman, et al., eds., 1983, pp. 55-64.

${ }^{23}$ Liepmann, H. W., and Roshko, A., Elements of Gasdynamics, Wiley, New York, 1957, pp. 329-333. 


\begin{tabular}{|c|c|c|c|c|}
\hline$j$ & Reaction & $\overline{a_{j}}$ & $\beta_{j}$ & $\overline{E_{j}}$ \\
\hline 1 & $\mathrm{O}_{2}+\mathrm{H} \rightarrow \mathrm{OH}+\mathrm{O}$ & $2.00 \times 10^{14}$ & 0.00 & 70.30 \\
\hline 2 & $\mathrm{OH}+\mathrm{O} \rightarrow \mathrm{O}_{2}+\mathrm{H}$ & $1.46 \times 10^{13}$ & 0.00 & 2.08 \\
\hline 3 & $\mathrm{H}_{2}+\mathrm{O} \rightarrow \mathrm{OH}+\mathrm{H}$ & $5.06 \times 10^{4}$ & 2.67 & 26.30 \\
\hline 4 & $\mathrm{OH}+\mathrm{H} \rightarrow \mathrm{H}_{2}+\mathrm{O}$ & $2.24 \times 10^{4}$ & 2.67 & 18.40 \\
\hline 5 & $\mathrm{H}_{2}+\mathrm{OH} \rightarrow \mathrm{H}_{2} \mathrm{O}+\mathrm{H}$ & $1.00 \times 10^{8}$ & 1.60 & 13.80 \\
\hline 6 & $\mathrm{H}_{2} \mathrm{O}+\mathrm{H} \rightarrow \mathrm{H}_{2}+\mathrm{OH}$ & $4.45 \times 10^{8}$ & 1.60 & 77.13 \\
\hline 7 & $\mathrm{OH}+\mathrm{OH} \rightarrow \mathrm{H}_{2} \mathrm{O}+\mathrm{O}$ & $1.50 \times 10^{9}$ & 1.14 & 0.42 \\
\hline 8 & $\mathrm{H}_{2} \mathrm{O}+\mathrm{O} \rightarrow \mathrm{OH}+\mathrm{OH}$ & $1.51 \times 10^{10}$ & 1.14 & 71.64 \\
\hline 9 & $H+H+M \rightarrow H_{2}+M$ & $1.80 \times 10^{18}$ & -1.00 & 0.00 \\
\hline 10 & $H_{2}+M \rightarrow H+H+M$ & $6.99 \times 10^{18}$ & -1.00 & 436.08 \\
\hline 11 & $\mathrm{H}+\mathrm{OH}+\mathrm{M} \rightarrow \mathrm{H}_{2} \mathrm{O}+\mathrm{M}$ & $2.20 \times 10^{22}$ & -2.00 & 0.00 \\
\hline 12 & $\mathrm{H}_{2} \mathrm{O}+\mathrm{M} \rightarrow \mathrm{H}+\mathrm{OH}+\mathrm{M}$ & $3.80 \times 10^{23}$ & -2.00 & 499.41 \\
\hline 13 & $O+O+M \rightarrow O_{2}+M$ & $2.90 \times 10^{17}$ & -1.00 & 0.00 \\
\hline 14 & $O_{2}+M \rightarrow O+O+M$ & $6.81 \times 10^{18}$ & -1.00 & 496.41 \\
\hline 15 & $\mathrm{H}+\mathrm{O}_{2}+\mathrm{M} \rightarrow \mathrm{HO}_{2}+\mathrm{M}$ & $2.30 \times 10^{18}$ & -0.80 & 0.00 \\
\hline 16 & $\mathrm{HO}_{2}+\mathrm{M} \rightarrow \mathrm{H}+\mathrm{O}_{2}+\mathrm{M}$ & $3.26 \times 10^{18}$ & -0.80 & 195.88 \\
\hline 17 & $\mathrm{HO}_{2}+\mathrm{H} \rightarrow \mathrm{OH}+\mathrm{OH}$ & $1.50 \times 10^{14}$ & 0.00 & 4.20 \\
\hline 18 & $\mathrm{OH}+\mathrm{OH} \rightarrow \mathrm{HO}_{2}+\mathrm{H}$ & $1.33 \times 10^{13}$ & 0.00 & 168.30 \\
\hline 19 & $\mathrm{HO}_{2}+\mathrm{H} \rightarrow \mathrm{H}_{2}+\mathrm{O}_{2}$ & $2.50 \times 10^{13}$ & 0.00 & 2.90 \\
\hline 20 & $\mathrm{H}_{2}+\mathrm{O}_{2} \rightarrow \mathrm{HO}_{2}+\mathrm{H}$ & $6.84 \times 10^{13}$ & 0.00 & 243.10 \\
\hline 21 & $\mathrm{HO}_{2}+\mathrm{H} \rightarrow \mathrm{H}_{2} \mathrm{O}+\mathrm{O}$ & $3.00 \times 10^{13}$ & 0.00 & 7.20 \\
\hline 22 & $\mathrm{H}_{2} \mathrm{O}+\mathrm{O} \rightarrow \mathrm{HO}_{2}+\mathrm{H}$ & $2.67 \times 10^{13}$ & 0.00 & 242.52 \\
\hline 23 & $\mathrm{HO}_{2}+\mathrm{O} \rightarrow \mathrm{OH}+\mathrm{O}_{2}$ & $1.80 \times 10^{13}$ & 0.00 & -1.70 \\
\hline 24 & $\mathrm{OH}+\mathrm{O}_{2} \rightarrow \mathrm{HO}_{2}+\mathrm{O}$ & $2.18 \times 10^{13}$ & 0.00 & 230.61 \\
\hline 25 & $\mathrm{HO}_{2}+\mathrm{OH} \rightarrow \mathrm{H}_{2} \mathrm{O}+\mathrm{O}_{2}$ & $6.00 \times 10^{13}$ & 0.00 & 0.00 \\
\hline 26 & $\mathrm{H}_{2} \mathrm{O}+\mathrm{O}_{2} \rightarrow \mathrm{HO}_{2}+\mathrm{OH}$ & $7.31 \times 10^{14}$ & 0.00 & 303.53 \\
\hline 27 & $\mathrm{HO}_{2}+\mathrm{HO}_{2} \rightarrow \mathrm{H}_{2} \mathrm{O}_{2}+\mathrm{O}_{2}$ & $2.50 \times 10^{11}$ & 0.00 & -5.20 \\
\hline 28 & $\mathrm{OH}+\mathrm{OH}+\mathrm{M} \rightarrow \mathrm{H}_{2} \mathrm{O}_{2}+\mathrm{M}$ & $3.25 \times 10^{22}$ & -2.00 & 0.00 \\
\hline 29 & $\mathrm{H}_{2} \mathrm{O}_{2}+\mathrm{M} \rightarrow \mathrm{OH}+\mathrm{OH}+\mathrm{M}$ & $2.10 \times 10^{24}$ & -2.00 & 206.80 \\
\hline 30 & $\mathrm{H}_{2} \mathrm{O}_{2}+\mathrm{H} \rightarrow \mathrm{H}_{2}+\mathrm{HO}_{2}$ & $1.70 \times 10^{12}$ & 0.00 & 15.70 \\
\hline 31 & $\mathrm{H}_{2}+\mathrm{HO}_{2} \rightarrow \mathrm{H}_{2} \mathrm{O}_{2}+\mathrm{H}$ & $1.15 \times 10^{12}$ & 0.00 & 80.88 \\
\hline 32 & $\mathrm{H}_{2} \mathrm{O}_{2}+\mathrm{H} \rightarrow \mathrm{H}_{2} \mathrm{O}+\mathrm{OH}$ & $1.00 \times 10^{13}$ & 0.00 & 15.00 \\
\hline 33 & $\mathrm{H}_{2} \mathrm{O}+\mathrm{OH} \rightarrow \mathrm{H}_{2} \mathrm{O}_{2}+\mathrm{H}$ & $2.67 \times 10^{12}$ & 0.00 & 307.51 \\
\hline 34 & $\mathrm{H}_{2} \mathrm{O}_{2}+\mathrm{O} \rightarrow \mathrm{OH}+\mathrm{HO}_{2}$ & $2.80 \times 10^{13}$ & 0.00 & 26.80 \\
\hline 35 & $\mathrm{OH}+\mathrm{HO}_{2} \rightarrow \mathrm{H}_{2} \mathrm{O}_{2}+\mathrm{O}$ & $8.40 \times 10^{12}$ & 0.00 & 84.09 \\
\hline 36 & $\mathrm{H}_{2} \mathrm{O}_{2}+\mathrm{OH} \rightarrow \mathrm{H}_{2} \mathrm{O}+\mathrm{HO}_{2}$ & $5.40 \times 10^{12}$ & 0.00 & 4.20 \\
\hline 37 & $\mathrm{H}_{2} \mathrm{O}+\mathrm{HO}_{2} \rightarrow \mathrm{H}_{2} \mathrm{O}_{2}+\mathrm{OH}$ & $1.63 \times 10^{13}$ & 0.00 & 132.71 \\
\hline
\end{tabular}

Table 1: Nine species, thirty-seven step reaction mechanism for hydrogen/oxygen/argon mixture ${ }^{20}$ also utilized by Fedkiw, et al. ${ }^{15}$. Units of $a_{j}$ are in appropriate combinations of $\mathrm{cm}$, mole, $s$, and $K$ so that $\dot{\omega}_{i}$ has units of mole $/ \mathrm{cm}^{3} / s$; units of $E_{j}$ are $k J /$ mole. Third body collision efficiencies with $M$ are $f_{H_{2}}=1.00$, $f_{\mathrm{O}_{2}}=0.35$, and $f_{\mathrm{H}_{2} \mathrm{O}}=6.5$. 